\title{
When is a Ketogenic Diet Ketogenic? Comment on "Satiating Effect of a Ketogenic Diet and Its Impact on Muscle Improvement and Oxidation State in Multiple Sclerosis Patients, Nutrients 2019, 11, 1156"
}

\author{
Rainer Johannes Klement $(\mathbb{D}$ \\ Department of Radiotherapy and Radiation Oncology, Leopoldina Hospital Schweinfurt, Robert-Koch-Straße 10, \\ 97422 Schweinfurt, Germany; rainer_klement@gmx.de; Tel.: +49-9721-7202761
}

Received: 1 July 2019; Accepted: 9 July 2019; Published: 15 August 2019

check for updates

Keywords: Ketogenic diet; Multiple sclerosis

\section{Dear Editor,}

Benlloch et al. recently published an article titled "Satiating Effect of a Ketogenic Diet and Its Impact on Muscle Improvement and Oxidation State in Multiple Sclerosis Patients" [1]. The authors showed that a Mediterranean diet supplemented with $2 \times 30 \mathrm{~mL}$ coconut oil daily led to significant increases of satiety at lunch and dinner and improvements of body composition in 27 multiple sclerosis patients. The authors attributed these changes mainly to elevations of the ketone body beta-hydroxybutyrate (BHB) (see their Figure 2). However, I doubt that this conclusion is supported by the data because the study unfortunately suffers from methodological problems.

1. The title and premise of the study are misleading, as the putative ketogenic diet (KD) did not conform to the typical definition of a KD based on its macronutrient compositions [2,3]. It provided $40 \%$ energy from carbohydrates and only $40 \%$ from fat, which would be too high and low, respectively, to induce nutritional ketosis, defined as serum beta-hydroxybutydare (BHB) levels exceeding $0.5 \mathrm{mmol} / \mathrm{L}[4,5]$.

2. The authors tried to induce ketogenesis by providing $30 \mathrm{~mL}$ coconut oil for breakfast and $30 \mathrm{~mL}$ for lunch each day. Hence, the term "ketogenic" diet might in principle be justified. However, the data cannot provide such justification because not even one postprandial measurement was undertaken to measure the putative increase in postprandial BHB concentration. Vandenberghe et al. [6] showed that $20 \mathrm{~mL}$ of coconut oil provided together with a mixed meal (breakfast) did not significantly stimulate ketosis-only when given without an additional meal did coconut oil induce a mild $(<0.5 \mathrm{mmol} / \mathrm{L})$ increase in $\mathrm{BHB}$ concentrations.

3. Patients were advised to eat five meals daily. This tended to minimize intermittent fasting periods during the day and counteracted the entry into a postabsorptive state in which insulin levels are minimized.

4. Indeed, the fasting serum BHB concentrations measured after 4 months were only $0.1 \pm 0.1 \mathrm{mmol} / \mathrm{L}$. Such levels are not unusual after an overnight fast on any diet. Although nominally significantly higher than pre-intervention BHB concentrations $(0.06 \pm 0.0 .4 \mathrm{mmol} / \mathrm{L})$, the $p$-value of 0.045 was not corrected for multiple testing and even if taken at face value offers only weak evidence against the null hypothesis of no pre-post difference [7].

Another dissonant point is that the authors referred to the BHB transporters MCT1 and MCT4 as medium-chain triglyceride transporters instead of the correct notation: monocarboxylate/ monocarboxylic acid transporters [8]. While I compliment the authors for their efforts to help multiple 
sclerosis patients by combining two beneficial concepts-those of a Mediterranean diet and ketosissuch a combination has been proposed before as the "Spanish Ketogenic Mediterranean Diet" which provides $<30 \mathrm{~g}$ carbohydrates per day and hence fits the common perception of a KD much better [9].

Conflicts of Interest: The authors have no conflict of interest.

\section{References}

1. Benlloch, M.; López-Rodríguez, M.M.; Cuerda-Ballester, M.; Drehmer, E.; Carrera, S.; Ceron, J.J.; Tvarijonaviciute, A.; Chirivella, J.; Fernández-García, D.; de la Rubia Ortí, J.E. Satiating Effect of a Ketogenic Diet and Its Impact on Muscle Improvement and Oxidation State in Multiple Sclerosis Patients. Nutrients 2019, 11, 1156. [CrossRef]

2. Westman, E.C.; Mavropoulos, J.; Yancy, W.S.; Volek, J.S. A Review of Low-carbohydrate Ketogenic Diets. Curr. Atheroscler. Rep. 2003, 5, 476-483. [CrossRef] [PubMed]

3. Kossoff, E.H.; Hartman, A.L. Ketogenic diets: New advances for metabolism-based therapies. Curr. Opin. Neurol. 2012, 25, 173-178. [CrossRef] [PubMed]

4. Miller, V.J.; Villamena, F.A.; Volek, J.S. Nutritional Ketosis and Mitohormesis: Potential Implications for Mitochondrial Function and Human Health. J. Nutr. Metab. 2018, 2018, 5157645. [CrossRef] [PubMed]

5. Harvey, C.J.D.C.; Schofield, G.M.; Williden, M. The use of nutritional supplements to induce ketosis and reduce symptoms associated with keto-induction: A narrative review. PeerJ 2018, 6, e4488. [CrossRef] [PubMed]

6. Vandenberghe, C.; St-Pierre, V.; Pierotti, T.; Fortier, M.; Castellano, C.-A.; Cunnane, S.C. Tricaprylin Alone Increases Plasma Ketone Response More Than Coconut Oil or Other Medium-Chain Triglycerides: An Acute Crossover Study in Healthy Adults. Curr. Dev. Nutr. 2017, 1, e000257. [CrossRef] [PubMed]

7. Held, L. An objective Bayes perspective on $p$-values. Biom. J. 2017, 59, 886-888. [CrossRef] [PubMed]

8. Pérez-Escuredo, J.; Van Hée, V.F.; Sboarina, M.; Falces, J.; Payen, V.L.; Pellerin, L.; Sonveaux, P. Monocarboxylate transporters in the brain and in cancer. Biochim. Biophys. Acta Mol. Cell Res. 2016, 1863, 2481-2497. [CrossRef] [PubMed]

9. Pérez-Guisado, J.; Muñoz-Serrano, A.; Alonso-Moraga, Á. Spanish Ketogenic Mediterranean diet: A healthy cardiovascular diet for weight loss. Nutr. J. 2008, 7, 30. [CrossRef] [PubMed]

(C) 2019 by the author. Licensee MDPI, Basel, Switzerland. This article is an open access article distributed under the terms and conditions of the Creative Commons Attribution (CC BY) license (http://creativecommons.org/licenses/by/4.0/). 